\title{
URUGUAY 2016: MAYORÍAS PARLAMENTARIAS EN JAQUE Y DESAFÍOS DE REVISIÓN PARA SOSTENER EL MODELO
}

\author{
Uruguay 2016: Parliamentary Majority Under Risk \\ and Challenges to Sustain the Model
}

\author{
JUAN A. BOGLIACCINI \\ Universidad Católica del Uruguay \\ ROSARIO QUEIROLO \\ Universidad Católica del Uruguay
}

\begin{abstract}
RESUMEN
Este artículo analiza los eventos políticos, económicos, sociales y de economía política ocurridos en Uruguay durante 2016, proveyendo un conjunto de información relevante y consistente con anteriores números de este anuario. El 2016 es un año clave en la política uruguaya dado que una serie de eventos de carácter político y económico ponen en tensión al gobernante Frente Amplio, tal vez por primera vez desde que está en el gobierno. En primer lugar, el Frente Amplio pierde la mayoría parlamentaria en la cámara de diputados, lo que plantea un escenario completamente nuevo para el gobierno. En segundo lugar, el déficit fiscal alcanza un $4 \%$ del PIB a diciembre de 2016, siendo el mayor déficit en más de dos décadas. Esto plantea un desafío tanto para el gobierno, como para la propia fuerza de izquierda, en términos de acuerdos sobre el programa de gobierno. En tercer lugar, el presidente Vázquez recibe durante 2016 más desaprobaciones a su gestión que aprobaciones, algo inédito desde 2005. En cuarto lugar, el fuerte desprestigio sufrido por la figura del vicepresidente Sendic acrecienta las tensiones políticas dentro de la coalición gobernante. Estamos ante el fin de una luna de miel de dos lustros del Frente Amplio (FA).
\end{abstract}

Palabras clave: Uruguay, Frente Amplio, mayoría parlamentaria, política económica

\begin{abstract}
This article analyzes the political, economic, and social events that occurred in Uruguay in 2016. This was a key year in Uruguayan politics given that a series of political and economic challenges put the ruling Frente Amplio (FA) under real strain, perhaps for the first time since it came to office. First, the Frente Amplio lost its parliamentary majority in the Chamber of Deputies, which created a completely new scenario for the government. Second, the fiscal deficit reached 4\% of GDP in December 2016: the largest deficit in more than two decades. This poses a challenge to both the government and its leftist agenda. Third, public approval of President Vaìquez has declined dramatically, leading to a situation in which more Uruguayans disapprove of their President than support him-something that has not happened since 2005. Finally, the accusations that led Vice-President Sendic to resign increased political tensions within the ruling coalition. This constellation of factors suggests that the extended honeymoon the Frente Amplio had enjoyed is now over.
\end{abstract}




\section{INTRODUCCIÓN}

El año 2016 fue el segundo de la segunda administración Vázquez, y también fue el año en que se terminó la luna de miel del Frente Amplio después de gobernar por dos períodos consecutivos (2005-2009, 2010-2014) y de haber empezado su tercer período de gobierno. La literatura sobre ciclos de gobierno insiste en que los primeros cien días de un presidente son su luna de miel, lo que suele llamarse en la disciplina el "honeymoon effect". Durante estos primeros cien días, los presidentes son más exitosos en lograr que el Parlamento apruebe sus leyes, a la vez que cuentan con mayor apoyo de la ciudadanía, que en el resto de su mandato. La magnitud de ese efecto de luna de miel depende del diseño institucional, de si el presidente cuenta con mayoría parlamentaria o el apoyo electoral con que haya llegado al gobierno. Evidencia de este efecto existe tanto para Estados Unidos (Edwards 1985; Beckmann y Godfrey2007) como para América Latina (Alemán y Calvo 2008), aunque sobre nuestra región la evidencia es menos conclusiva (Alemán y Navia 2009). En este artículo planteamos que el 2016 es el final de la luna de miel del gobierno del Frente Amplio, pero no solo de este tercer gobierno sino de los tres períodos en que ha gobernado el país.

El segundo año del tercer gobierno del Frente Amplio claramente queda fuera de cualquier efecto de luna de miel. Existen al menos cuatro eventos clave, de distinta índole, que inciden en este cambio: la desaceleración económica, la pérdida de la mayoría parlamentaria, el hecho de que Vázquez reciba durante 2016 más desaprobaciones a su gestión que aprobaciones, y el desprestigio de la figura del vicepresidente Raúl Sendic, el cual es concomitante a una serie de investigaciones sobre hechos de posible corrupción, o al menos mala gestión, que se inician en este tercer período. ${ }^{1}$

En primer lugar, en materia fiscal, el gobierno comenzó a implementar un plan de "consolidación fiscal" tendiente a reducir un déficit que en diciembre llegó al 4\%, el mayor en el último cuarto de siglo. La tercera administración consecutiva del Frente Amplio heredó de la administración Mujica (2010-2014) una situación de amplio deterioro fiscal, el cual se vuelve más preocupante en un contexto de fuerte desaceleración económica en el marco del fin del "boom de los commodities". Las exportaciones uruguayas de bienes tuvieron una reducción anual de 7,3\% respecto de 2015, año en el que a su vez habían caído en un 11,6\% respecto de 2014 (Uruguay XXI 2015, 2016). La combinación de estos factores, junto con una incipiente tendencia al alza del desempleo y una creciente conflictividad en los ámbitos tripartitos de negociación salarial en la nueva ronda de acuerdos, amenaza con erosionar, o al menos estancar los importantes logros en materia de desarrollo social y económico conseguidos en la última década y media. 
En segundo lugar, el partido de gobierno perdió la mayoría parlamentaria en la Cámara de Diputados por primera vez desde que asumió el gobierno en 2005, debido al alejamiento de las filas partidarias del diputado Gonzalo Mujica, ${ }^{2}$ electo por el Movimiento de Participación Popular (Espacio 609, uno de los grupos con mayor representación parlamentaria dentro del Frente Amplio). Este hecho-de no mediar una renuncia a la banca por parte del diputado disidente y junto con otros movimientos en filas de la oposición- plantea hacia adelante un escenario diferente para las estrategias de gobierno y oposición, donde uno y otros tendrán que acordar la agenda de gobierno. Sin embargo, aunque la pérdida de la mayoría parlamentaria pareciera dar a priori mayor poder a la oposición, su fragmentación en cinco partidos -incluyendo el nuevo Partido de la Gente- aumenta los costos de coordinación para elevar el precio que el gobierno necesita pagar para obtener el último voto.

En tercer lugar, Tabaré Vázquez, que terminó su primer mandato (2005-2009) con una aprobación de su gestión de $72 \%$ — la más alta de un presidente uruguayo desde que se hacen mediciones de este tipo—, obtiene durante el 2016 un balance negativo de su trabajo como presidente: las desaprobaciones a su gestión son más que las aprobaciones.

En cuarto y último lugar, el Poder Ejecutivo recibió un fuerte golpe asociado con el desprestigio de la figura del vicepresidente de la República, Raúl Sendic. Este aspecto ha tenido un fuerte impacto en la imagen del Frente Amplio de la última década, un capital político importante que el partido de gobierno cultiva y utiliza. Durante la primera mitad del año se instaló una polémica pública por la existencia o no del título profesional del vicepresidente; polémica que, asociada con una investigación sobre supuestas irregularidades durante la gestión de Raúl Sendic como presidente de la empresa petrolera del Estado, terminó por debilitar fuertemente su figura como posible presidenciable en las elecciones de 2019.

Estos cuatro factores implican un cambio que impacta negativamente en las condiciones favorables que el Frente Amplio tuvo desde su llegada al gobierno en 2005. Sin embargo, no todas las noticias fueron negativas para el gobierno en 2016. En materia de exportaciones, los últimos dos meses del año presentaron una mejora del 5\% respecto de igual período en 2015, lo que permite arriesgar que el segundo semestre de 2016 puede haber sido un punto de inflexión en términos del enlentecimiento económico. En materia de inversiones, el gobierno entró en negociaciones decisivas durante 2016 con la empresa UPM para la instalación de una tercera planta de celulosa en el país — segunda de este inversor-, lo que supondría un empuje decisivo a la economía nacional, desacelerada desde 2015. Sin embargo, la buena noticia no viene exenta de desafíos, dado que como contrapartida a la inversión, la empresa finlandesa puso como requisito una

Nota de los editores: El senador Gonzalo Mujica renunció a su banca el 13 de setiembre de 2017, con lo que le devolvió las mayorías parlamentarias al FA. 
fuerte inversión en infraestructura vial y ferroviaria por parte del gobierno, lo que supondrá una prioridad presupuestaria para el resto del período que deberá ser integrada al proceso de baja del déficit fiscal antes mencionado.

En materia de política internacional, dos hechos destacan como los más importantes del año. Por un lado, y tras 50 años, Uruguay vuelve a integrar el Consejo de Seguridad de Naciones Unidas como miembro no permanente. ${ }^{3}$ En segundo lugar, el gobierno firmó un Tratado de Libre Comercio (TLC) con Chile, el segundo que Uruguay firma tras el que acordara con México en 2003.

El artículo se organiza de la siguiente forma. En primer lugar se describen los principales cambios ocurridos en 2016 en la situación laboral, fiscal y macroeconómica del país, los cuales definen condiciones estructurales que limitan el margen de acción del gobierno. La tercera sección describe las elecciones internas del Frente Amplio, las cuales conformaron el acto eleccionario más relevante de 2016, y analiza los principales cambios en la interna de los partidos políticos uruguayos y el impacto que estas alteraciones implican para la coordinación entre Poder Ejecutivo y Legislativo. La sección cuarta se dedica al análisis de lo ocurrido con el Poder Ejecutivo, los cambios sucedidos en el gabinete ministerial y la aprobación popular que recibe el Presidente. La quinta sección describe los impactos que se produjeron en el Poder Legislativo con la pérdida de la mayoría absoluta para el gobierno y otros movimientos realizados en el interior de los partidos de la oposición. Por último desarrollamos algunas conclusiones.

\section{PANORAMA LABORAL, FISCAL Y MACROECONÓMICO}

En 2016 cerró con un déficit fiscal del 4\% del producto bruto interno (PIB), el mayor en el último cuarto de siglo. En relación con este problema y la escalada inflacionaria del lustro anterior-Figura 1-, el año 2016 marca el inicio de la estrategia de la administración Vázquez para moderar dichas tendencias y, eventualmente, revertirlas. El Ministerio de Economía presentó un paquete de medidas que denominó de "Consolidación Fiscal", mientras que la oposición cataloga como de "Ajuste Fiscal". Entre las medidas tomadas se destacan el aumento del impuesto a la renta de las personas físicas (IRPF) a partir de la segunda franja (salarios nominales en torno de USD 1.000 y superiores); un incremento en todas las tarifas públicas de alrededor del $8 \%$ (aunque se hace apenas por debajo de la inflación anual para el período enero-diciembre, que fue de $8,1 \%$ ) y la moderación del gasto por la vía de recortes o su dilación para los últimos años del período. Aunque estas medidas entran en efecto en 2017, por lo que su efectividad no puede ser evaluada aún, el gobierno logra contener la inflación ya en el último trimestre del $2016 .{ }^{4}$ 
Figura 1. Evolución del panorama fiscal e inflacionario

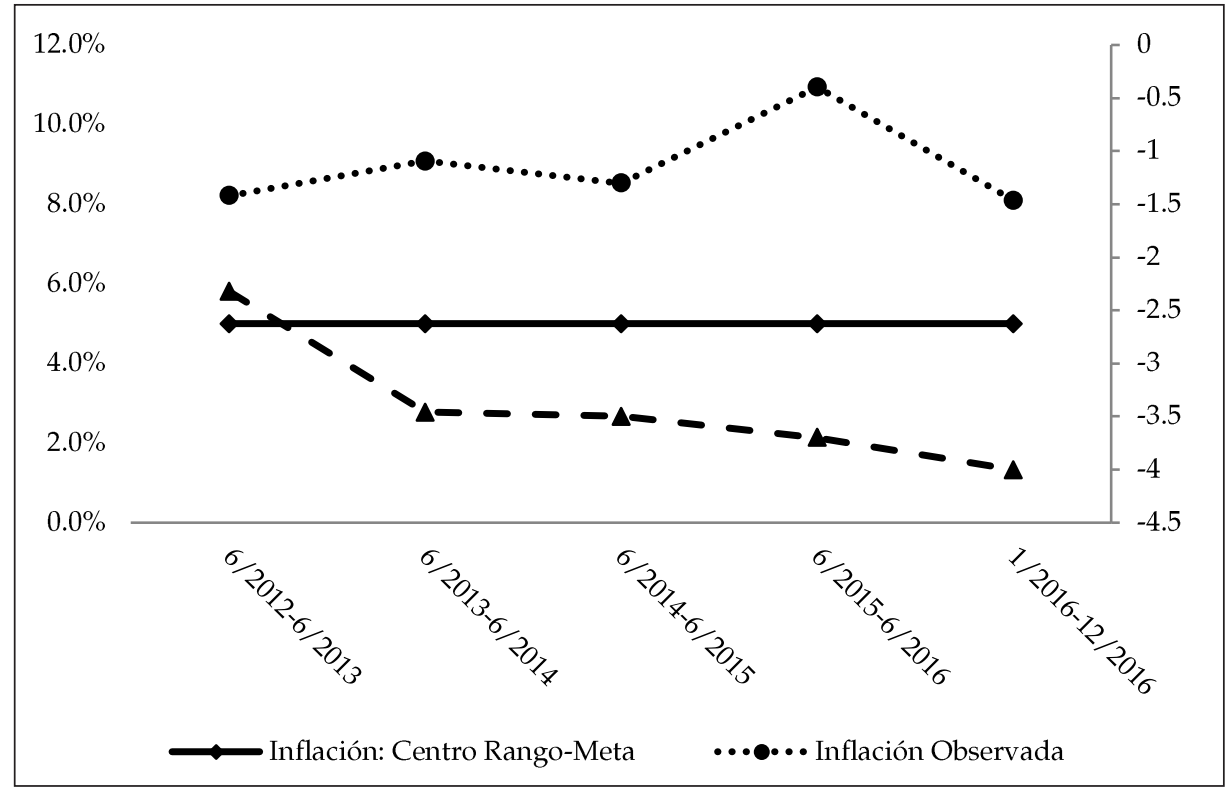

Fuente: Datos del Banco Central del Uruguay. Nota: Balance fiscal en eje secundario

La desaceleración económica también fue percibida por los ciudadanos durante el 2016. El desempleo abandonó un período de cuatro años de estabilidad en torno al 6,5\% entre 2011 y 2014 para subir a 7,5\% en 2015 y continuar con dicha tendencia en 2016 al llegar al 8,1\% en diciembre (INE 2016). Esto es esperable dado que el énfasis puesto por el gobierno en la recuperación del balance fiscal, sumado al instrumento de negociación centralizada de salarios que otorga cierta inercia a la suba de ellos, hacen del nivel de empleo — fundamentalmente en el sector transable exportador- la principal válvula de ajuste del proceso (Iversen 2005). La Figura 2 muestra la evolución en el Índice de Confianza del Consumidor, que alcanza los 40 puntos en mayo, el más bajo de los últimos 10 años; aunque recuperándose en diciembre de 2016 hasta alcanzar el nivel de marzo de 2015, el mejor en los últimos dos años. 
Figura 2. Índice de Confianza del Consumidor (2007-2016)

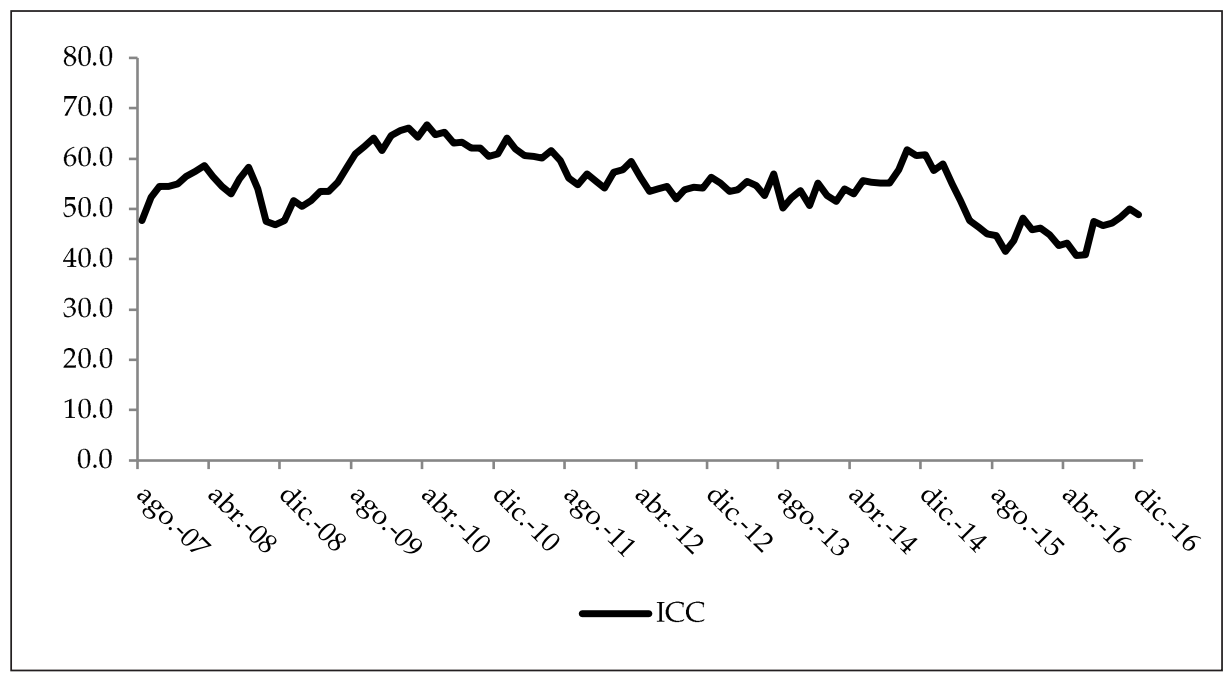

Fuente: Cátedra SURA de Confianza Económica, Universidad Católica del Uruguay, Equipos Consultores

La sexta ronda de negociación salarial (2015-2016) dejó al descubierto posibles problemas de diseño institucional del modelo de negociación colectiva y de los instrumentos que el gobierno tiene para lograr armonizar la política laboral con otras áreas, en particular la política macroeconómica. En especial nos referimos al desafío para moderar la demanda por mayor salario, específicamente de los sectores no transables, durante períodos de enlentecimiento económico. En sistemas de negociación salarial con centralización intermedia, como es el caso de Uruguay, ya que se negocia por grupos de actividad en base a un calendario que se extiende al menos 12 meses en cada ronda, los sindicatos enfrentan un problema de acción colectiva respecto de la exigencia de aumentos salariales desmedidos y su potencial efecto en el desempleo (Swenson 1989, Iversen 1999).

Este problema, cuyo riesgo aumenta en períodos de enlentecimiento económico o de escasa mejora en niveles de productividad como ocurrió en 2016, se refleja en los datos de la Tabla 1. Los consensos tripartitos disminuyen notoriamente entre las rondas cinco (2013) y seis (2015-2016). Mientras que el patrón de la negociación de la ronda de 2013 guarda estrecha relación con las rondas anteriores, en la sexta ronda se observan dos nuevos patrones. En primer lugar, crece consistentemente el porcentaje de ámbitos en que el gobierno vota junto a los empresarios, intentando mantener el incremento salarial dentro de la pauta original. ${ }^{5}$ En segundo lugar, y con tendencia creciente en el año 2016,

\footnotetext{
El gobierno elabora una pauta de negociación salarial para cada ronda, que establece mínimos y máximos que este está dispuesto a votar. Al ser un sistema de negociación tripartito, el gobierno garantiza el cumplimiento de la pauta siempre que su voto sea definitorio. Sin embargo, acuerdos bipartitos entre empresarios y trabajadores no necesitan homologación por parte del Ministerio de Trabajo y pueden, potencialmente, circunvalar la pauta.
} 
empresarios y trabajadores votan juntos por sobre la pauta estipulada por el gobierno. Mientras que en el primer caso el gobierno logra controlar el incentivo a la militancia salarial por parte de los sindicatos, en el segundo no lo logra.

Tabla 1. Modalidad de resolución de negociación en Consejos de Salarios, rondas 5 y 6

\begin{tabular}{|c|c|c|c|c|c|c|}
\hline Fecha & $\begin{array}{c}\text { Ámbitos } \\
\mathrm{N}^{\circ}\end{array}$ & $\begin{array}{c}\text { Consenso } \\
(\%)\end{array}$ & $\begin{array}{l}\text { Empresa- } \\
\text { rios y traba- } \\
\text { jadores (\%) }\end{array}$ & $\begin{array}{c}\text { Poder } \\
\text { Ejecutivo } \\
\text { y empresa- } \\
\text { rios (\%) }\end{array}$ & $\begin{array}{c}\text { Poder } \\
\text { Ejecutivo } \\
\text { y traba- } \\
\text { jadores } \\
(\%)\end{array}$ & $\begin{array}{l}\text { Decre- } \\
\text { tos }(\%)\end{array}$ \\
\hline $5^{\mathrm{a}}$ ronda oct $/ 13$ & 132 & 92 & 3 & 2 & 4 & 0 \\
\hline \multicolumn{7}{|l|}{$6^{\mathrm{a}}$ ronda } \\
\hline Semestre 2, 2015 & 64 & 64 & 11 & 22 & 0 & 3 \\
\hline Semestre 1, 2016 & 33 & 58 & 33 & 3 & 6 & 0 \\
\hline Semestre 2, $2016\left(^{* *}\right)$ & 43 & 70 & 28 & 0 & 2 & 0 \\
\hline Total $6^{\mathrm{a}}$ ronda & 140 & 64 & 21 & 11 & 2 & 2 \\
\hline
\end{tabular}

${ }^{* *}$ Convenios publicados hasta el 15/12/2016 en Ministerio de Trabajo y Seguridad Social Fuente: Instituto de Relaciones Laborales, Universidad Católica del Uruguay

El crecimiento de los consensos observado en el segundo semestre de 2016 responde, según Rodríguez et al. (2016), a la flexibilización de la pauta salarial ofrecida por el gobierno en noviembre en relación con los correctivos por inflación, principal disputa entre el gobierno y los trabajadores. A partir de la flexibilización, 30 convenios fueron consensuados, revirtiendo la tendencia hasta ese momento. De esta forma, el gobierno logra atenuar el efecto de la militancia salarial a partir de concesiones controladas (segunda mejor opción al no poder mantener las pautas originales).

La ronda 2015-2016 de negociación salarial, sin haber sido un fracaso, envía importantes señales que el gobierno deberá tornar en aprendizajes para poder sobrellevar instancias de negociación en el futuro que se realicen en contextos de crecimiento poco favorables. Mientras la pauta de correctivos por inflación originalmente propuesta por el gobierno fue acatada por la mayoría de ámbitos privados, en particular los sectores transables de la economía, en varios de los sectores no transables lograron negociar ajustes diferidos a un plazo menor -en el entorno de un año. Hubo incluso un caso extremo: la empresa estatal ALUR (Alcoholes del Uruguay), donde los correctivos por inflación se negociaron cada dos meses. Aunque este último convenio fue anulado por el Poder Ejecutivo y resultó incluso en la sustitución de sus autoridades, el problema queda en evidencia.

En primer lugar, en el ámbito macroeconómico, en particular de política monetaria, se debiera contar con mecanismos que refuercen la credibilidad del 
gobierno cuando este necesite establecer una política monetaria restrictiva. En el presente, el único mecanismo de credibilidad existente reside en la confianza en el respaldo que el Poder Ejecutivo le ofrezca a la autoridad monetaria. Sin embargo, este es un instrumento sumamente frágil. ${ }^{6}$ La falta de credibilidad de la autoridad monetaria en estos contextos arriesga picos inflacionarios y pérdida de control sobre el balance fiscal, particularmente en economías con fuerte peso del empleo estatal, como también es el caso de Uruguay. En este sentido y desde la década de 1990, varios países de Europa continental e incluso algunos escandinavos que se movieron desde modelos de negociación salarial centralizados a intermediamente centralizados, han establecido la independencia del Banco Central como mecanismo garante de la credibilidad de la autoridad monetaria (Soskice et al. 2000).

En segundo lugar y asociado a lo anterior, la incorporación de algún tipo de regla fiscal efectiva es otro instrumento útil para controlar gasto e incluso inflación salarial en los sectores no transables de la economía (Licandro 2000). En tercer lugar, el modo en que los diferentes ámbitos lograron negociar los correctivos por inflación en la ronda 2016, inicialmente diferidos a dos años por el gobierno, también sugiere discutir el rol que dichos sectores de la economía, en particular el sector público, debieran tener en la negociación salarial a fin de garantizar la sostenibilidad del modelo. En relación con el sector público en particular, una solución encontrada por diversos países de Europa Central y Escandinavia es fijar sus aumentos - dado que es un sector que tiene altos niveles de protección del empleo- en el promedio de los aumentos del resto de la economía, para facilitar que el ritmo al que los salarios aumentan esté primariamente ligado a aumentos en la productividad de los sectores transables de la economía (Iversen 1999, Soskicee Iversen 2000).

\section{ELECCIONES}

Durante 2016 no hubo elecciones de carácter nacional pero sí hubo elecciones internas dentro del Frente Amplio, y también sucedieron realineamientos importantes dentro del Partido Nacional y Partido Colorado, a la vez que surgió un nuevo partido político, el Partido de la Gente. En esta sección analizamos cada uno de estos acontecimientos.

\section{Las internas del Frente Amplio}

En la actualidad, el Frente Amplio es definido como el único partido orgánico de masas (Levitsky y Roberts 2011) de América Latina. A pesar de llevar tres 
períodos consecutivos al frente del gobierno nacional, no ha perdido sus características fundamentales como partido con una fuerte representación política tanto de militantes de base como de los sectores que lo componen (Rosenblatt et al. 2016). Esta representación y participación se da a través de la integración de los sectores y militantes en el Plenario Nacional y los Plenarios Departamentales. Los integrantes de estos plenarios son electos en las elecciones internas del partido. En ellas votan los adherentes o cualquier persona con residencia uruguaya que tenga 16 años y más y decida adherirse en el momento de la votación. Si bien los resultados de estas elecciones internas del Frente Amplio son para elegir las autoridades del partido, y no son vinculantes para la definición de los candidatos a cargos de representación nacional o departamental (como sí lo son las elecciones internas que se celebran obligatoriamente a nivel nacional para todos los partidos políticos durante el año electoral), sí definen la relación de fuerzas dentro del conglomerado. Tener el control del partido es importante porque el Frente Amplio es quien negocia con el gobierno, con los sectores y con los militantes, establece los lineamientos programáticos y define posiciones políticas (Rosenblatt et al. 2016).

Los resultados de las elecciones internas del Frente Amplio de 2016 no introdujeron ningún cambio en la relación de fuerza que ya existía dentro del partido, sino que confirmaron el liderazgo del bloque más radical. Actualmente el Frente Amplio cuenta con 29 sectores, de los cuales 10 obtuvieron representación parlamentaria en la elección de 2014, y pueden ser agrupados en tres bloques. El sector más radical a la izquierda del espectro ideológico, compuesto por el Movimiento de Participación Popular (MPP) del expresidente José Mujica, el Partido Comunista, Casa Grande (sector de la senadora Contanza Moreira) y Compromiso Frenteamplista (sector del vicepresidente Raúl Sendic), obtuvo la mayoría de los votos en la elección interna (47,3\%). De esta manera consolidó su liderazgo, a pesar de que no pudo coordinar para convertir a uno de sus dos candidatos (Alejandro Sánchez y Roberto Conde) en presidente del partido. Precisamente el hecho de que el sector más radical presentara dos candidatos permitió al Frente Líber Seregni, liderado por el actual ministro de economía y exvicepresidente Danilo Astori, obtener la presidencia del partido. Este bloque alcanzó el 19,8\% de los votos, y a pesar de tener menos poder en la interna del partido, logró coordinar con el tercer bloque, el Partido Socialista (12,5\% de los votos en la interna), para poner a su candidato Javier Miranda como presidente. Esto deja al Frente Amplio con una marcada división interna. Por un lado, un presidente moderado y cercano al presidente Vázquez; por otro lado, el resto de la dirección más radical. Si bien esta situación no es nueva, dado que ya existía bajo la presidencia de Mónica Xavier, representa un desafío en momentos como la definición de la Rendición de Cuentas y en particular en un contexto de necesaria austeridad fiscal como en el próximo 2017. 


\section{Realineamientos postelecciones en el Partido Nacional}

El año 2016 fue también un año bisagra para uno de los dos grandes sectores del Partido Nacional, el ala wilsonista-Alianza Nacional-, que fuera el sector derrotado en las elecciones internas del partido en el año 2014. La clave para comprender los eventos en este sector es el desafío al líder del mismo - Jorge Larrañaga- por parte de senadores e intendentes (gobernadores). Larrañaga, que es líder del sector desde el período electoral de 1999, lleva dos derrotas consecutivas a manos del otro gran sector del partido, posicionado más a la derecha —el herrerismo—, en los procesos eleccionarios de 2009 y 2014.

El desafío al liderazgo de Larrañaga con miras al proceso eleccionario del año 2019 proviene de dos subsectores diferentes. Por un lado, la senadora Verónica Alonso, mujer joven con un perfil independiente, que ha crecido en forma sostenida en las urnas, se presenta como la principal desafiante al liderazgo de Larrañaga. La senadora Alonso fue electa para la Cámara de Diputados en 2009 por el herrerismo, moviéndose en el período eleccionario de 2014 al sector de Larrañaga, cuando resultó electa al Senado al ocupar el tercer lugar en la lista.

Por otro lado, cuatro de los ocho intendentes electos por el sector de Larrañaga en las elecciones de 2014 -Adriana Peña (Lavalleja), Sergio Botana (Cerro Largo), Dardo Sánchez (Treinta y Tres) y Enrique Antía (Maldonado)—, junto al intendente blanco independiente de Tacuarembó —Eber Da Rosa-, han avanzado en la segunda mitad de 2016 en la idea de formar un sector separado de Alianza Nacional para las elecciones de 2019. Alianza Nacional sufrió además la deserción de un diputado a manos del nuevo Partido de la Gente.

En los últimos días de diciembre, Larrañaga envió a unos 2.500 dirigentes del sector un documento orientado a renovar la estructura del sector. Dicho documento, denominado "Nuestro Futuro Juntos", plantea de forma poco concreta diversos mecanismos posibles de agrupar a los líderes y otros de fuera del sector en pos de generar una nueva alternativa al herrerismo para el próximo proceso eleccionario de 2019. No obstante este proceso de realineamiento político recién comienza en 2017, su evolución será clave para conocer si el herrerismo, liderado por el senador Lacalle Pou, tendrá un competidor fuerte en las elecciones primarias de 2019 o logrará un triunfo sencillo frente a una oposición dividida y atomizada. Por su parte, el otro sector del Partido Nacional, el herrerismo, no parece tener problemas en la definición de su liderazgo. Luis Lacalle Pou aparece como el claro líder del sector, lo que ha llevado a que durante 2016 se haya concentrado en ser la oposición del gobierno, en algunos momentos incluso simulando lo que podría ser un gabinete en las sombras.

\section{Atomización del Partido Colorado}

Las elecciones de 2014 dieron otro duro golpe al Partido Colorado, con apenas un $12,89 \%$ de los votos en la primera vuelta, solo cuatro senadores y trece 
diputados, con un saldo negativo de un senador y cuatro diputados menos respecto del período anterior. Sumado a este magro resultado electoral, entre diciembre de 2015 y agosto de 2016 el partido sufrió la deserción de un senador y un diputado a manos del entonces Partido de la Concertación, ahora Partido de la Gente. Este contexto ha favorecido el desafío al liderazgo del senador Pedro Bordaberry.

El sector de Bordaberry - Vamos Uruguay-, que obtuviera tres senadores y once diputados, perdió entre 2015 y 2016 todos los diputados por Montevideo (4), más los dos legisladores que pasaron al Partido de la Gente. Tres nuevos sectores se perfilaron a partir de estas deserciones: Revolución Batllista, liderado por la diputada Valentina Rapella; Batllismo Abierto, liderado por el diputado Ope Pasquet; y Batllistas Orejanos, liderado por el diputado Fernando Amado. La poca disciplina del sector Vamos Uruguay puede tener una explicación en que él mismo ordenó su lista a la Cámara de Diputados de acuerdo con los votos obtenidos en la elección interna, sin permitir ningún tipo de acumulación de votos y aplicando la representación proporcional integral, lo cual disminuye el poder del líder del sector y aumenta el de los representantes (Cantero y Guedes 2016). El liderazgo del propio Bordaberry ha marcado cierto declive en 2016, pues ya no es visto como la carta renovadora del partido por varios dirigentes. ${ }^{7}$

Por su parte, el sector minoritario del partido - Propuesta Battlista (PROBA)—, liderado por el senador Amorín Battle, sufrió la deserción de sus dos diputados - Tabaré Viera y Conrado Rodríguez-, quienes junto al intendente de Rivera, Marne Osorio, fundaron el sector Espacio Abierto, con la intención de unirse con el nuevo sector de Ope Pasquet. De este modo, el único parlamentario que le queda al PROBA es su propio líder, Amorín Batlle. La separación de Viera y Osorio cobra relevancia dado que ambos son los líderes colorados en el departamento de Rivera, único gobierno departamental en manos del Partido Colorado.

Por tanto, el 2016 presenta una atomización en la estructura de un partido en proceso de decadencia. De todos modos, es factible esperar un proceso de realineamiento de estos nuevos pequeños sectores en los próximos dos años, de cara a las elecciones internas de 2019.

\section{Surgimiento del Partido de la Gente}

El Partido de la Gente se conformó como tal el 7 de noviembre de 2016 bajo el liderazgo de Edgardo Novick para competir en las elecciones de 2019-2020. Novick fue candidato a intendente de Montevideo en las elecciones de 2015 por el Partido de la Concertación, y esa elección lo llevó a transformarse en una

A fines de abril de 2017, en momentos que se imprime este artículo, Pedro Bordaberry anunció su intención de no ser candidato a la presidencia en las elecciones nacionales de 2019, lo que consolida el escenario de reagrupamiento de sectores dentro del Partido Colorado. 
figura de alcance nacional. El surgimiento del Partido de la Concertación fue el resultado de la coordinación electoral del Partido Colorado y Partido Nacional con el objetivo de derrotar al Frente Amplio en la elección departamental de Montevideo (por mayores detalles ver López-Cariboni y Moraes 2014). Si bien ese objetivo fracasó, ya que el Frente Amplio volvió a ganar el gobierno de Montevideo con el 55,7\% de los votos, mientras que la Concertación obtuvo el $41,6 \%$, el resultado electoral tuvo como efecto secundario la creación de la figura política de Edgardo Novick, un colorado independiente que hasta el momento no había tenido actividad política electoral de relevancia.

Novick compitió como candidato a intendente de Montevideo como uno de los tres candidatos que presentó el Partido de la Concertación ${ }^{8}$ y obtuvo la mayoría absoluta de los votos dentro del partido $(63,4 \%)$ con un discurso basado en la importancia de realizar una gestión eficiente del gobierno y dejar de lado a las ideologías, ya sean de derecha o izquierda. Como afirman Pérez y Piñeiro (2016), la aparición de Novick como figura política es algo nuevo para Uruguay, ya que no es usual que las carreras políticas se hagan fuera de los partidos establecidos.

Con este antecedente exitoso en las elecciones departamentales, durante el bienio 2015-2016 Novick buscó adhesiones entre legisladores de los otros partidos y logró el apoyo de un senador (Bianchi) y un diputado (Facello) del Partido Colorado, así como un diputado del Partido Nacional (Peña). A estos legisladores hay que sumarles los ediles de Montevideo electos por el Partido de la Concertación. Si bien ninguno de estos legisladores o ediles fueron electos por el Partido de la Gente, actualmente apoyan a Novick, dando al partido representación parlamentaria a pesar de no haber competido en las elecciones nacionales de 2014. Hasta el momento, el nuevo partido político ha reclutado sus líderes entre viejos dirigentes de otros partidos y no entre cuadros nuevos. El Partido de la Gente se presenta como un partido más allá de las ideologías, centrando su discurso en lograr una administración eficiente. ${ }^{9}$ Su actuación parlamentaria es aún escasa tanto a nivel nacional como departamental, pero destacan el acuerdo alcanzado entre Novick y el intendente de Montevideo Daniel Martínez en enero de 2016, para que la Junta Departamental aprobara un fideicomiso denominado "Fondo Capital", de 94 millones de dólares, para obras públicas. Como contraparte, el gobierno departamental se comprometió a reducir el gasto del gobierno departamental.

\section{PODER EJECUTIVO}

El 2016 presentó desafíos importantes para el gobierno de Tabaré Vázquez. En primer lugar, la evaluación dela gestión del presidente, que a fines del 2015 había

Los otros dos candidatos por el Partido de la Concertación fueron Ricardo Rachetti por el Partido Colorado y Álvaro Garcé por el Partido Nacional.

Su programa de gobierno puede encontrarse en el siguiente enlace: http:/ /www.novick.com.uy/edgardo/ Ver además El Observador (2016). 
comenzado a tener un balance negativo - los juicios negativos sobre la forma en que el presidente estaba conduciendo el país superaban a los positivos-, se volvió más crítica. La Figura 3 indica que las aprobaciones bajan durante el primer semestre del año, y si bien la tendencia parece detenerse e incluso revertirse en el segundo semestre, las aprobaciones continúan en torno de un tercio de la población.

Figura 3. Aprobación de la gestión del presidente Vázquez

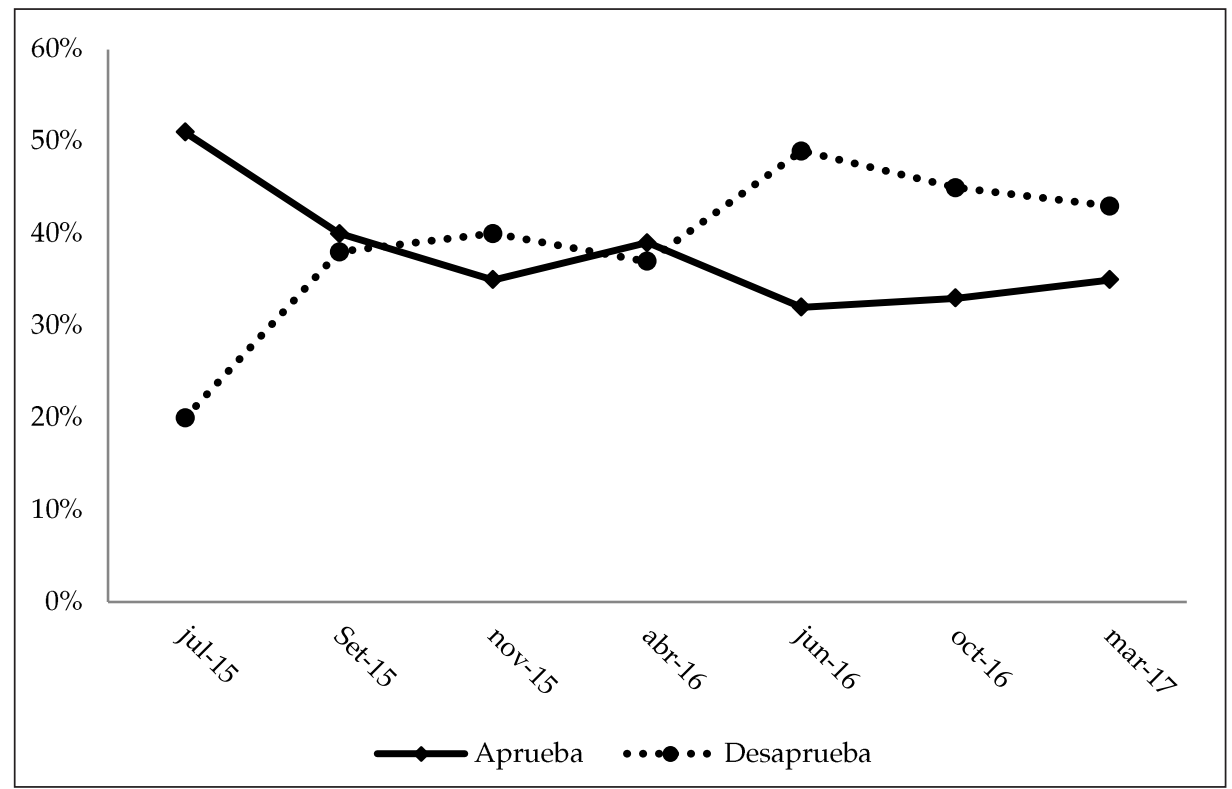

Fuente: CIFRA

Es razonable que la evaluación de la gestión del presidente empeore con el correr de los meses, siendo que esto le sucedió anteriormente a José Mujica (Moraes et al. 2012), y al mismo Vázquez durante su primer gobierno (Garcé 2010); y con el correr de los años cuando un partido permanece en el poder. El tercer gobierno del Frente Amplio, segundo de Tabaré Vázquez, comenzó con un nivel de apoyo popular significativamente menor que el primero. Mientras Vázquez comenzó su primer mandato con el $64 \%$ de la población apoyando su gestión, en el segundo el nivel de apoyo inicial descendió 11 puntos porcentuales (53\%) (ver Figura 4). A pesar de este descenso, el caudal de apoyo con que iniciaron todos los gobiernos del Frente Amplio es mayor que el recibido por cualquier otro gobierno desde la transición a la democracia; incluso el de Jorge Batlle —el primer presidente que llega al cargo después de una segunda vuelta electoral一, que a pocos meses de estar en el gobierno tenía una aprobación récord para los antecedentes del país (49\% de aprobación en abril) (González y Queirolo 2000). 
Figura 4. Aprobación de la gestión del Presidente (promedio 2005-2015 y serie 2016)

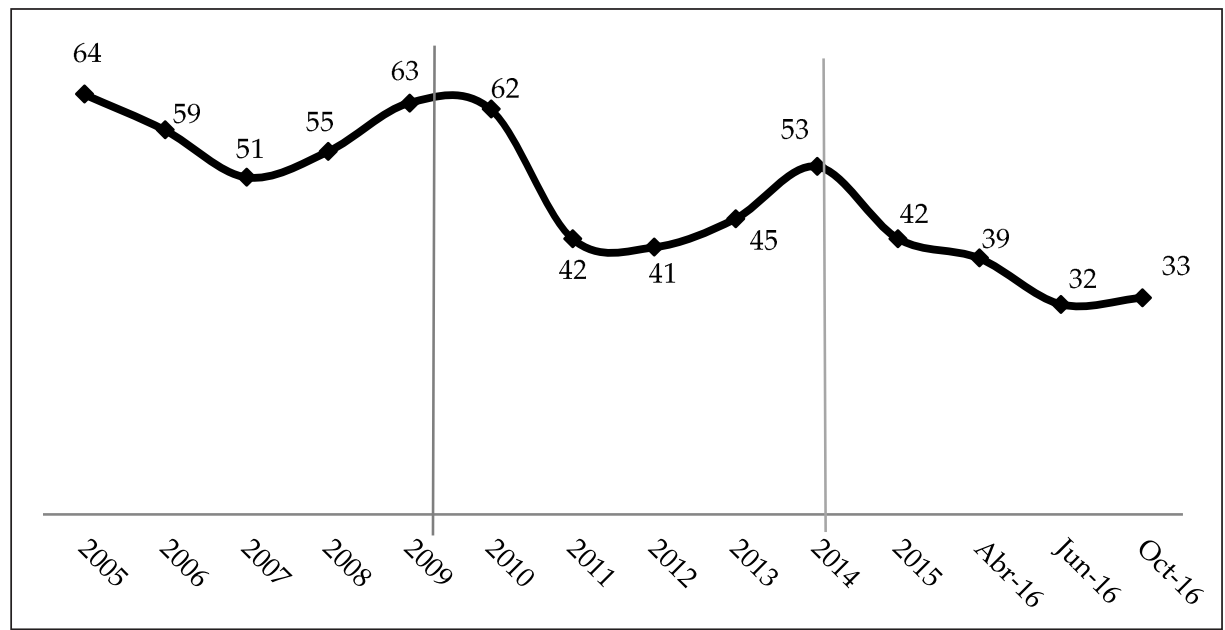

Fuente: CIFRA

Esta evaluación negativa del desempeño de Tabaré Vázquez a cargo del Ejecutivo se vio acompañada del desmoronamiento de la figura del vicepresidente Raúl Sendic (ver Figura 5). El sector de Raúl Sendic fue el más votado dentro del Frente Amplio en las elecciones internas de 2014. Esto lo llevó a ocupar el cargo de vicepresidente en la fórmula con Vázquez, y a ser visto como posible candidato a Presidente para la elección del 2019. Sin embargo, en febrero de 2016 se generó una controversia porque el título de Licenciado con que el vicepresidente se presentaba, incluso en la página web de su sector político, no existía. Si bien Sendic ha consistentemente declarado que realizó cursos de genética en la Universidad de la Habana, no existe un título de licenciado. El problema de la titulación, sumado a las dudas en torno de su gestión al frente de ANCAP, genera la pérdida de popularidad. 
Figura 5. Popularidad del vicepresidente Raúl Sendic

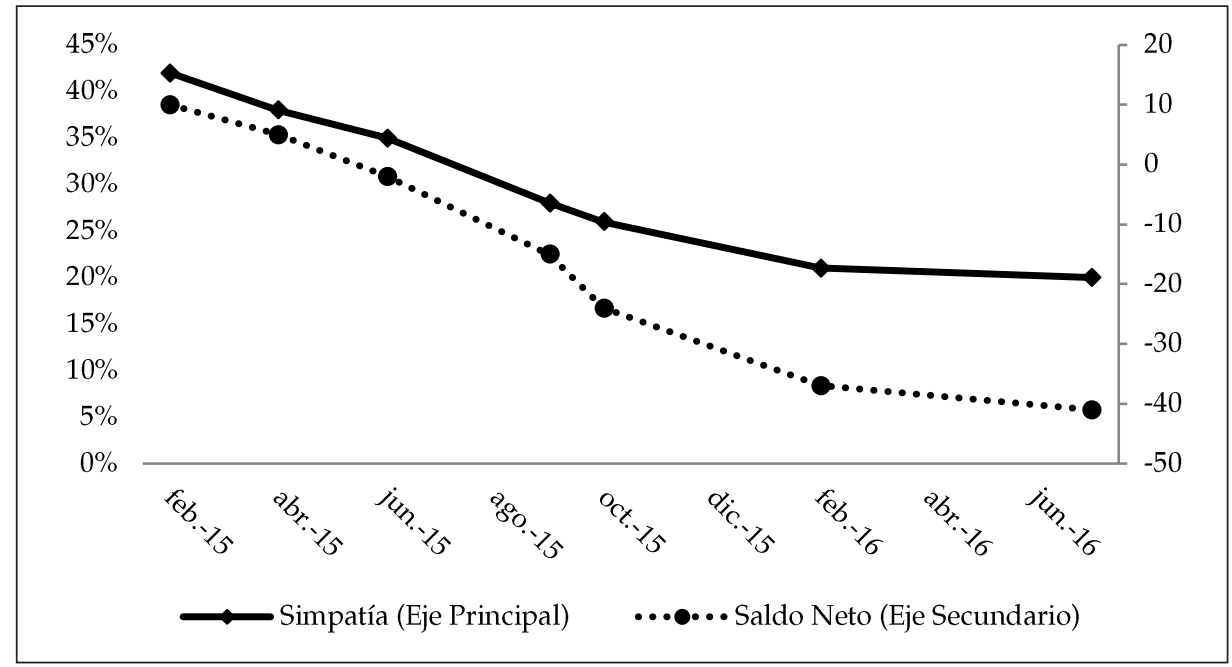

Fuentes: Equipos Mori / El País http:/ /www.elpais.com.uy/informacion/imagen-sendic-se-desmorona-encuesta.html; UYPress - http://www.uypress.net/auc.aspx?70308

Más allá de la aprobación del Presidente y los eventos relacionados con el Vicepresidente, el 2016 fue un año que no presentó cambios importantes para el gabinete ministerial. La única variación significativa en la cartera por causas naturales y no políticas fue la muerte de Eleuterio Fernández Huidobro, ministro de Defensa Nacional. Fernández Huidobro asumió la dirección del Ministerio por primera vez en 2011, bajo el gobierno de José Mujica, y fue renovado en su cargo con la llegada de Vázquez a la presidencia. A su muerte, el subsecretario Jorge Menéndez asume como ministro.

Al inicio del gobierno de Vázquez, se especuló con un cambio amplio en su gabinete a mitad de mandato, ya que el promedio de edad de los ministros de gobierno, al asumir su cargo en 2015, era de 65,2 años. Sin embargo, esto no ha sucedido hasta el momento.

En términos de la producción legislativa del Poder Ejecutivo, los gobiernos suelen tener el pico de actividad, presentando proyectos de leyes para ser aprobados por el Parlamento, durante los primeros años del período (Chasquetti 2014). En Uruguay, debido a que el Poder Ejecutivo tiene iniciativa parlamentaria en todos los temas e incluso en algunos tiene "iniciativa privativa", es decir que sólo el Ejecutivo puede enviar proyectos, es muy alto el porcentaje de proyectos a estudio del Parlamento que son iniciados por el Ejecutivo (Chasquetti 2007; Chasquetti y Guedes 2011; Buquet y Luján 2011; López Cariboni y Moraes 2014; Pérez y Piñeiro 2016).

Durante 2016, el Ejecutivo de Vázquez siguió la tendencia histórica y fue dominante frente al Legislativo en proporción de leyes enviadas respecto de las sancionadas: 94 presentadas por el Ejecutivo versus 23 presentadas por el 
Parlamento. Esto es a pesar de haber presentado el Ejecutivo menos proyectos de ley que el Legislativo (ver Tabla 2), lo que también muestra la preeminencia del Ejecutivo en el pautado de la agenda legislativa en este período.

Tabla 2. Proyectos presentados y leyes sancionadas en la $48^{\circ}$ Legislatura (según iniciativa por Poder)

\begin{tabular}{lcccc}
\hline & 2015 & 2016 & 2015 & 2016 \\
\cline { 2 - 5 } & \multicolumn{2}{c}{ Proyectos presentados } & \multicolumn{2}{c}{ Leyes sancionadas } \\
\hline \multirow{2}{*}{ Poder Legislativo } & 248 & 187 & 7 & 23 \\
& $67 \%$ & $67 \%$ & $14 \%$ & $20 \%$ \\
\multirow{2}{*}{ Poder Ejecutivo } & 121 & 92 & 42 & $94^{*}$ \\
& $33 \%$ & $33 \%$ & $86 \%$ & $80 \%$ \\
Total & 369 & 279 & 49 & 117 \\
& $100 \%$ & $100 \%$ & $100 \%$ & $100 \%$ \\
\hline
\end{tabular}

Fuente: Datos del Programa de Estudios Parlamentarios. ICP-FCS-UdelaR

*Las leyes sancionadas con iniciativa del Poder Ejecutivo en 2016 son más que los proyectos presentados en el 2016, esto se debe a que incluyen proyectos desarchivados que fueron presentados en el período de gobierno anterior y aquellos que siendo aprobados en 2016, fueron presentados en 2015.

Otro indicador para conocer el grado de avance del Poder Ejecutivo es el informe de cumplimiento de metas realizado por UyCheck. El segundo año de gobierno presenta un $24,17 \%$ de cumplimiento en las metas declaradas tanto en su programa de gobierno como en el discurso de asunción de mando del presidente Vázquez el 1 de marzo de 2010. Las dos áreas donde el logro es mayor es derechos humanos (56\%) y seguridad ciudadana y vial $(42,2 \%)$ (UYCheck 2017). Estos porcentajes son acumulados, incluyen todas las políticas implementadas desde que el gobierno asumió en 2015. Dado que esta medición en el país lleva pocos años, no contamos con la posibilidad de comparar estos números con los de administraciones anteriores.

Finalmente, el año 2016 presenta solo dos interpelaciones ministeriales, una al Ministro del Interior (Eduardo Bonomi) y la otra al Ministro de Economía (Danilo Astori), ambas resueltas con voto de satisfacción por parte del parlamento. ${ }^{10}$ Ambas interpelaciones pueden interpretarse de forma diferente. Los Ministros de Economía y Finanzas han sido los miembros del gabinete más interpelados entre 1985 y 2016, en 34 oportunidades. En este sentido, una nueva interpelación

10 Los Ministros de Estado son nombrados por el Presidente de la República con venia parlamentaria. El parlamento puede convocarlos para pedir explicaciones sobre temas de interés particular, debiendo hacer un voto de satisfacción o insatisfacción. Esto puede derivar en una moción de voto de censura de un ministro. Ver artículos 147 y 148 de la Constitución de la República para mayor detalle sobre estos procedimientos. Ver página de Programa de Estudios Parlamentarios para detalle de procesos de interpelaciones y votos de censura para el período 1985-2016: https: / / parlamentosite.wordpress.com/interpelaciones / 
en el marco de un proceso de "consolidación/ajuste" fiscal resulta previsible. ${ }^{11}$ La interpelación al ministro Bonomi, en cambio, debe ser interpretada desde otra óptica por dos motivos: en primer lugar, dicho ministro ha sido el más interpelado desde la vuelta a la democracia en 1985, tuvo 7 interpelaciones en 11 años en el cargo, lo que refleja un conflicto entre gobierno y oposición de largo aliento en materia de seguridad pública. En segundo lugar, el problema de la inseguridad pública ha pasado a los primeros lugares de preocupación de la opinión pública en la última década, por lo que es un tema reiterado en las agendas políticas de los diferentes partidos, principalmente de oposición. ${ }^{12}$

\section{PODER LEGISLATIVO}

El año 2016 ha sido un parteaguas en términos de composición parlamentaria desde que el Frente Amplio llegó al gobierno en 2005. Por primera vez desde entonces, el partido de gobierno ha perdido la mayoría absoluta en la Cámara de Diputados tras el alejamiento del diputado del Movimiento de Participación Popular, Gonzalo Mujica. Dicho alejamiento es esperable que traiga aparejado un cambio en las estrategias parlamentarias de gobierno y oposición a partir del año 2017.

El alejamiento de Mujica se dio a partir de desavenencias internas del Frente Amplio sobre la forma de procesar las incipientes investigaciones sobre irregularidades en diferentes entes y programas del gobierno. En particular, su disposición a votar una investigación parlamentaria sobre la gestión del Fondo para el Desarrollo (FONDES) y la desavenencia con la negativa del Frente Amplio de apoyar la instalación de una investigación parlamentaria sobre los negocios con Venezuela — que lo llevaron a ser citado por el tribunal de Ética del Frente Amplio - detonaron la partida (Pereyra 2016).

En este sentido, la pérdida de la mayoría parlamentaria ocurre en un contexto, durante 2015 y 2016, de fuerte revisión de diversas gestiones denunciadas por mala práctica o corrupción en los primeros dos gobiernos del Frente Amplio. Desde este punto de vista, el promedio de pre-investigadoras formadas en el Parlamento por año, para los primeros dos años de la presente administración, es la mayor desde que el Frente Amplio es gobierno (Figura 6).

11 Datos obtenidos del Programa de Estudios Parlamentarios (s/f). Recuperados el 1 de agosto de 2017 de: https://parlamentosite.wordpress.com/interpelaciones/

12 Datos obtenidos del Programa de Estudios Parlamentarios (s/f). Recuperados el 1 de agosto de 2017 de: https://parlamentosite.wordpress.com/interpelaciones/ 
Figura 6. Investigadoras y Pre-investigadoras Parlamentarias por período de gobierno

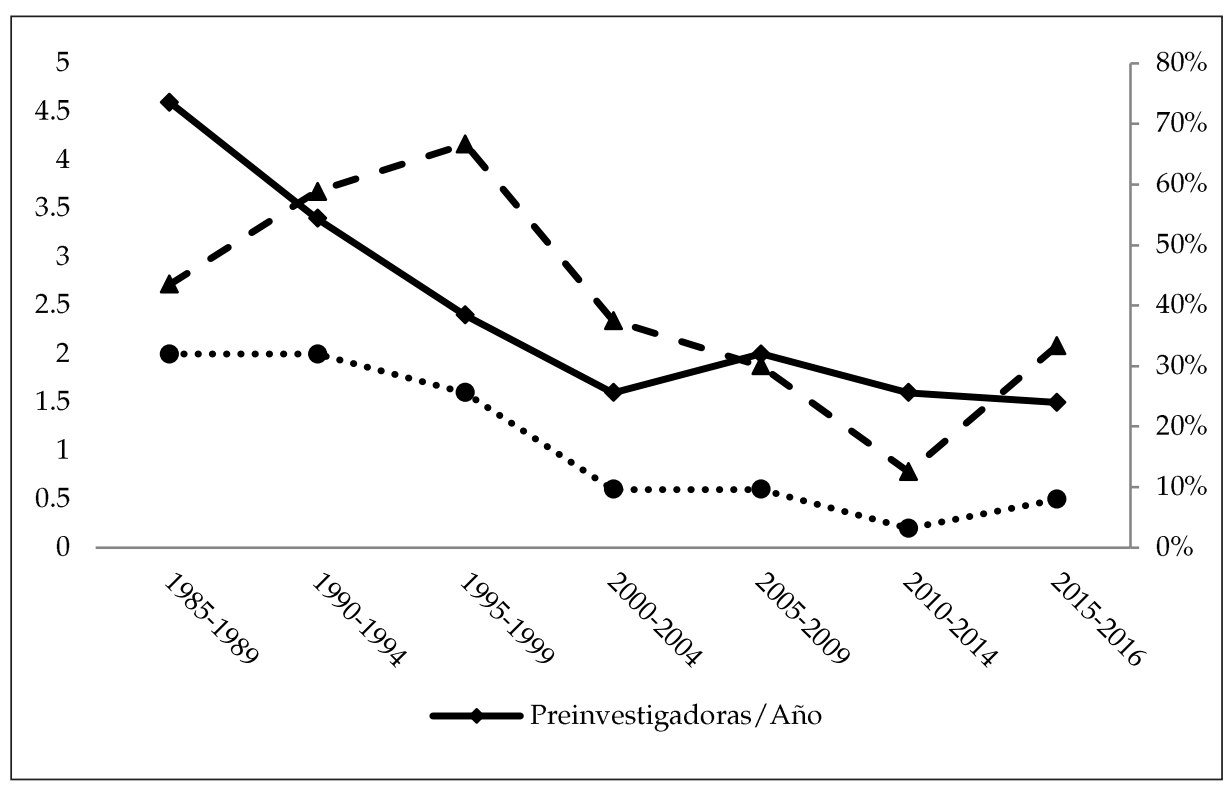

Fuente: Elaboración propia, basados en datos del Programa de Estudios Parlamentarios. Universidad de la República. https://parlamentosite.wordpress.com/las-comisiones-investigadoras-en-uruguay/

El otro aspecto importante a destacar en la vida parlamentaria durante 2016 es la consolidación de un proceso de "pasaje" de diputados y senadores electos por los partidos tradicionales hacia el movimiento político de Edgardo Novick. Durante 2016 dicho movimiento integró un senador —Daniel Bianchi, del Partido Colorado- (diciembre de 2015), y dos diputados —Guillermo Facello, del Partido Colorado y Daniel Peña, del Partido Nacional-, en mayo y diciembre respectivamente.

Este movimiento en filas de la oposición otorga voluntad parlamentaria a un movimiento no electo para tal fin en las elecciones nacionales de 2014originalmente el Partido de la Concertación y, desde 2016, el Partido de la Gente. No obstante la creación del Frente Amplio en 1971 y la separación del Nuevo Espacio de este en 1988 son antecedentes similares desde el punto de vista del cambio de lealtad partidaria, el actual proceso se diferencia en que ocurre tempranamente en el período de gobierno, en la segunda legislatura, y en que no se basa en el movimiento de fracciones o sectores, sino de voluntades individuales desde diversos sectores y partidos.

El alejamiento del diputado Mujica tendrá potenciales efectos en la actividad parlamentaria futura, pero fundamentalmente en relación con las Rendiciones de Cuentas a partir de 2017. Esto puede favorecer a los sectores moderados del Frente Amplio en términos de decisiones sobre presupuesto y gastos, en un contexto de ajuste o consolidación fiscal, según gobierno u oposición lo 
denominan. En términos más generales, los partidos de la oposición tienen una puerta abierta a partir de ahora para negociar mayorías a cambio de introducir intereses propios en la agenda de gobierno.

La atomización parlamentaria como resultado del citado movimiento de figuras desde los partidos tradicionales al Partido de la Gente, la permanencia del propio Gonzalo Mujica como independiente en el parlamento y el hecho de que la pérdida de mayoría se da a partir de la falta de un solo voto, hacen suponer que el costo que el Frente Amplio debe asumir para obtener el voto que precisa para recuperar la mayoría parlamentaria no será necesariamente alto.

En definitiva, la trascendencia de este proceso es incierta pero es razonable especular que en un contexto en que el Frente Amplio necesita un voto para lograr la mayoría en diputados estos nuevos actores parlamentarios disminuyen la "capacidad de chantaje" (Sartori 2005) de los cuatro partidos de oposición con representación parlamentaria. Por otro lado, este cambio aumenta los costos de coordinación a la interna de la coalición de izquierda, al tener que negociar necesariamente el último voto con un actor externo (Baron y Farejohn 1987, 1989).

A su vez, este escenario permite al nuevo Partido de la Gente, potencialmente, aumentar su capital político de cara a las elecciones nacionales de 2019. En términos históricos, los eventos del año 2016 permiten confirmar la tendencia al alza de partidos con representación parlamentaria y número efectivo de partidos al inicio de las legislaturas de 2009 y 2004 respectivamente (Figura 7).

Figura 7. Partidos con representación parlamentaria y número efectivo de partidos

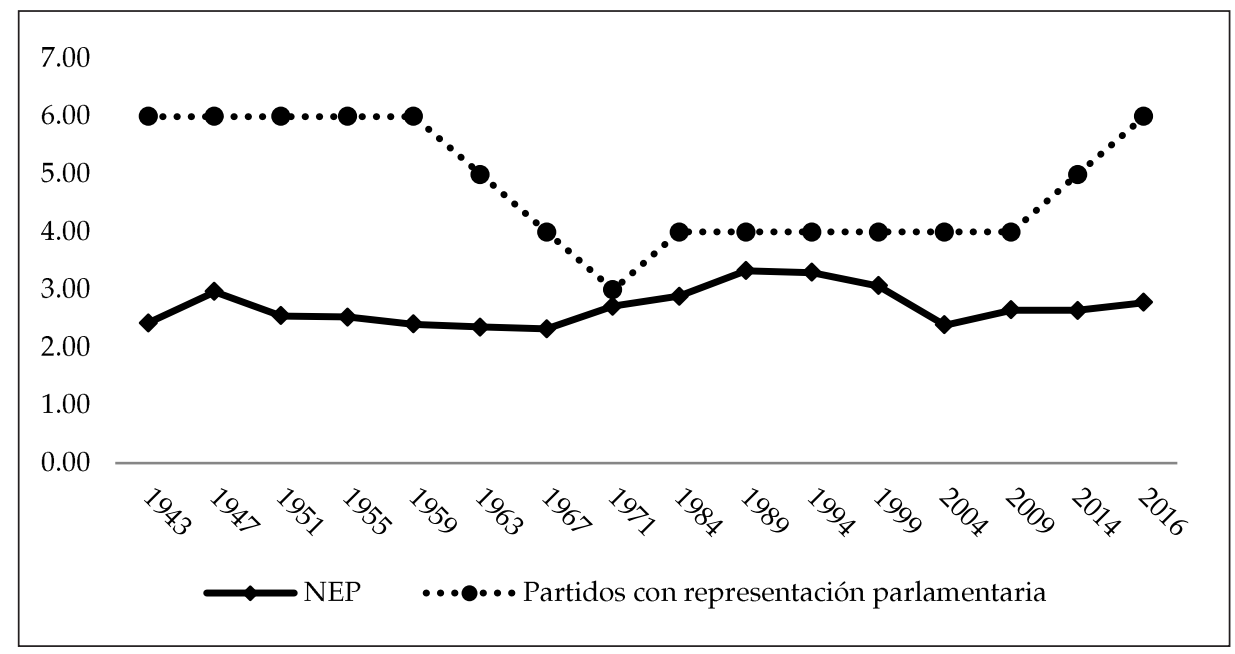

Fuente: Elaboración propia en base a datos del Banco de Datos de la Facultad de Ciencias Sociales, UdelaR Nota: El año 2016 incluye al Partido de la Concertación/Partido de la Gente. No se cuenta al diputado independiente (Gonzalo Mujica, ex Frente Amplio) como partido. 
En conclusión, y de acuerdo con la literatura, es esperable que el Frente Amplio modere sus posturas en lo que resta de la legislatura al haberse hecho más débil su posición a partir de la pérdida de la mayoría absoluta en la Cámara de Diputados (McDonald y Budge 2005; Adams y Merrill 2009). Esta moderación puede incidir en la representación de preferencias, ya que los gobiernos que no tienen mayoría parlamentaria tienden a representar a un espectro más amplio de intereses existentes en la sociedad, que aquellos que cuentan con mayoría (Wlezien y Soroka 2007).

\section{CONCLUSIONES}

El año 2016 representa un año bisagra a partir de cambios en diversos aspectos importantes que hacen a la actuación del gobierno. En primer lugar, la consolidación/ajuste fiscal implicó un cambio importante en la dinámica presupuestaria, generando desafíos importantes para la búsqueda de acuerdos internos en la coalición de izquierda. Este cambio es el resultado de un deterioro fiscal progresivo heredado de la administración anterior en combinación con el fin del llamado "boom de los commodities". Sumado a esto, el Frente Amplio perdió la mayoría absoluta en Cámara de Diputados, lo que obliga a buscar acuerdos fuera del partido. Finalmente, y por razones diferentes, las figuras de Presidente y Vicepresidente sufrieron caídas importantes en la opinión pública. En el caso del Presidente, la caída sigue el patrón observado en las anteriores administraciones del Frente Amplio, aunque a un nivel menor de aprobación general. En el caso del Vicepresidente, un desprestigio de su figura política coadyuvó a robustecer una imagen deteriorada del Ejecutivo, al menos en el corto plazo, dado que a fines de 2016 la aprobación de la gestión del presidente Vázquez ha cesado de empeorar.

Algunos de los aspectos analizados en este artículo tendrán un impacto de corto plazo en el gobierno, mientras que otros podrán tornarse en desafíos importantes de cara al próximo período electoral. Mientras el desprestigio de la imagen del Vicepresidente, en tanto figura pública y posible presidenciable para el ciclo eleccionario de 2019, impacta negativamente, el Frente Amplio tiene otros posibles nombres en la mesa con capital político suficiente como para competir. La evaluación de la gestión del gobierno, atada a los problemas de inseguridad, educación y balance fiscal, evolucionarán de mejor o peor forma asociados fundamentalmente con el devenir económico del país durante el resto del período, dado que los problemas de inseguridad y educación no son pasibles de ser resueltos en el corto plazo, más allá de que el gobierno pueda mostrar avances específicos en ambos frentes.

La pérdida de mayoría parlamentaria, de no revertirse, pondrá un desafío hasta el final del período, aunque no solo para el Frente Amplio sino también para la oposición. El voto faltante para la mayoría abrirá una oportunidad a la oposición de capitalizar el efecto de nuevas estrategias de participación activa en la agenda 
política, no solo desde una posición de oposición pasiva y minoritaria. Esto, por supuesto, podrá beneficiar o perjudicar a los diferentes sectores según como se juegue en el nuevo escenario.

Por otro lado, la atomización de la oposición, con el surgimiento del Partido de la Gente y la aparente múltiple división interna del Partido Colorado, también podrán modificar el escenario electoral de estas fuerzas así como sus estrategias para mostrarse como alternativas viables de gobierno.

Finalmente, y desde una perspectiva estructural, el análisis de la ronda de negociación salarial 2016 dejó al descubierto potenciales falencias del modelo, que el Frente Amplio debiera atender para mejor garantizar su sostenibilidad en el largo plazo. Este aspecto es relevante dado que la consolidación del Frente Amplio como un partido de base trabajadora depende en gran medida de la salud y tamaño del movimiento sindical. En ese sentido, la dificultad para moderar la demanda sindical por mayor salario, en particular de los sectores no transables durante períodos de enlentecimiento económico, se presenta como un riesgo importante a ser atendido por el gobierno. Optar por reglas fiscales o una mayor independencia del Banco Central no son alternativas necesariamente equivalentes desde el punto de vista distributivo, y necesitan ser exploradas en detalle.

\section{REFERENCIAS}

Alemán, Eduardo y Ernesto Calvo.2008. "Analyzing Legislative Success in Latin America: The Case of Argentina." En New Voices in the Study of Democracy in Latin America, editado por Guillermo O'Donnell et al. Woodrow Wilson Center Reports on the Americas 19. Washington: Woodrow Wilson International Center for Scholars, 7-37.

Alemán, Eduardo y Patricio Navia. 2009. "Institutions and the Legislative Success of 'Strong' Presidents: An Analysis of Government Bills in Chile." The Journal of Legislative Studies 15(4): 401-419.

Adams, Jim y Samuel Merrill III. 2009.“Policy-seeking Parties in a Parliamentary Democracy with Proportional Representation: A Valence-uncertainty Model." British Journal of Political Science 39(03): 539-558.

Baron, David y John A. Ferejohn. 1989. "Bargaining in Legislatures." American Political Science Review 83(4):1181-1206.

Baron, David y John A. Ferejohn 1987."Bargaining and Agenda Formation in Legislatures." The American Economic Review 77(2):303-309.

Beckmann, Matthew y Joseph Godfrey.2007. "The Policy Opportunities in Presidential Honeymoons." Political Research Quarterly 60(2): 250-262.

Buquet, Daniel y Diego Luján. 2011. "El Poder Ejecutivo." En Política en tiempos de Mujica. En busca del rumbo, editado por Gerardo Caetano, María Ester Mancebo y JuanAndrés Moraes. Informe de Coyuntura No 10, Instituto de Ciencia Política, Montevideo: Estuario Editora, 183-193.

Cantero Piali, Fitzgerald y Alejandro Guedes. 2016. "Elecciones primarias y confección de hojas nacionales: el caso de Vamos Uruguay." En Permanencias, Transiciones y Rupturas. Elecciones en Uruguay 2014/15, editado por Adolfo Garcé y Niki Johnson. Montevideo: Instituto de Ciencia Política y Editorial Fin de Siglo, 285-298.

Chasquetti, Daniel. 2014. Parlamento y carreras legislativas en Uruguay: Un estudio sobre reglas, partidos y legisladores en las Cámaras. Montevideo: UdelaR. 
Chasquetti, Daniel. 2007.“Uruguay 2006: éxitos y dilemas del gobierno de izquierda.” Revista de Ciencia Política 27(1): 249-263.

Chasquetti, Daniel y Alejandro Guedes. 2011. “El primer año legislativo del gobierno de Mujica." En Política en tiempos de Mujica. En busca del rumbo, Informe de Coyuntura $\mathrm{N}^{\circ} 10$, Instituto de Ciencia Política. Estuario Editora, 183-202.

Edwards, George C. 1985. “Measuring Presidential Success in Congress: Alternative Approaches." Journal of Politics 47(2): 667-685.

El Observador. 2016, 7 de noviembre. “Novick registró el Partido de la Gente: 'Hoy es un día histórico'." El Observador. Recuperado el 1 de agosto de 2017 de http:/ / www.elobservador.com.uy/novick-registro-el-partido-la-gente-hoy-es-un-dia-historico-n995199

Garcé, Adolfo. 2010. "Uruguay 2009: de Tabaré Vázquez a José Mujica." Revista de Ciencia Política 30(2): 499-535.

Gonzalez, Luis E. y Rosario Queirolo. 2000. “Las elecciones nacionales del 2004: posibles escenarios." En Las elecciones 1999/2000. Montevideo: Instituto de Ciencia Política-UdelaR y Banda Oriental.

INE. 2016. Estadísticas varias. Recuperado el 1 de agosto de 2017 dehttp:/ / www.ine.gub.uy /

Iversen, Torben, 1999. Contested Economic Institutions: The Politics of Macroeconomics and Wage Bargaining in Advanced Democracies. New York: Cambridge University Press.

Iversen, Torben, 2005. Capitalism, Democracy and Welfare. New York: Cambridge University Press.

Levitsky, Steven y Kenneth Roberts 2011.TheResurgence of The Left in Latin America. Baltimore: Johns Hopkins University Press.

Licandro, Gerardo. 2000. "Las reglas de responsabilidad fiscal en el Uruguay." Documento de Trabajo del BCU 01/2000.

López Cariboni, Santiago y Juan Andrés Moraes. 2014. “Uruguay 2013: Un balance preelectoral." Revista de Ciencia Política 34(1): 293-313.

McDonald, Michael e Ian Budge. 2005. Elections, Parties, and Democracy: Conferring the Median Mandate. Oxford: Oxford University Press.

Moraes, Juan Andrés, Alejandro Guedes y Diego Lujan. 2012. “Uruguay:¿dónde está el piloto? A dos años de gobierno del Presidente Mujica (2010-2011)."Revista de Ciencia Política 32(1): 269-291.

Pereyra, Leonardo. 2016, diciembre 7. “Gonzalo Mujica se fue del Frente Amplio: 'Hoy es un día de quiebre'." El Observador. Recuperado el 1 de agosto de 2017 de http://www. elobservador.com.uy/gonzalo-mujica-se-fue-del-frente-amplio-hoy-es-un-dia-quiebre-n1008198

Pérez, Verónica y Rafael Piñeiro. 2016. “Uruguay 2015: Los desafíos de gobernar por izquierda cuando la economía se contrae." Revista de Ciencia Política 36(1): 339-363.

Programa de Estudios Parlamentarios (s/f). Recuperados el 1 de agosto de 2017 de: https:/ / parlamentosite.wordpress.com/interpelaciones/

Rodríguez, Juan Manuel et al. 2016. La sexta ronda de negociación salarial. Facultad de Ciencias Empresariales. Universidad Católica del Uruguay.

Rosenblatt, Fernando, Rafael Piñeiro y Verónica Pérez. 2016. “Internas del Frente Amplio: continuidad en un contexto de cambios." Revista Nueva Sociedad Digital. Recuperado el 1 de agosto de 2017 de http://nuso.org/articulo/internas-del-frente-amplio-continuidad-en-un-contexto-de-cambios/

Sartori, Giovanni. 2005.Partidos y sistemas de partidos. $2^{\text {da }}$ edición. Madrid: Alianza Editorial.

Soskice, David,Torben Iversen y Jonas Pontusson.2000. Unions, Employers, and Central Banks: Macroeconomic Coordination and Institutional Change in Social Market Economies. New York: Cambridge University Press.

Soskice, David y Torben Iversen. 2000. "The non neutrality of Monetary Policy with Large Price or Wage Setters." The Quarterly Journal of Economics 115(1): 265-284.

Swenson, Peter. 1989. Fair Shares. Ithaca: Cornell University Press. 
Uruguay XXI. 2016. "Informe anual de comercio exterior." Recuperado el 1 de agosto de 2017 de http://www.uruguayxxi.gub.uy/informacion/knowledge-base/informe-de-comercio-exterior-de-uruguay-2016/

Uruguay XXI. 2016. “Informe anual de comercio exterior." Recuperado el 1 de agosto de 2017 de http://www.uruguayxxi.gub.uy/informacion/wp-content/uploads/sites/9/2016 /01/Informe-Anual-Comercio-Exterior-2015.pdf

UYCheck 2017. "Informe del Dicho al Hecho 2017." Recuperado el 1 de agosto de 2017 de https://drive.google.com/file/d/0BzxjmZqoaxQmdzUyemFWY19RcDQ/view

Wlezien, Christopher y Stuart N. Soroka. 2007. "The Relationship between Public Opinion and Policy." En The Oxford Handbook of Political Behavior, editado por Russell Dalton y Hans-Dieter Klingemann. New York: Oxford University Press.

Juan A. Bogliaccini es profesor del Departamento de Ciencias Sociales y Políticas de la Universidad Católica del Uruguay. Se doctoró en el Departamento de Ciencia Política de la Universidad de Carolina del Norte en Chapel Hill (Estados Unidos). Su trabajo ha sido publicado en Economics \& Politics, Latin American Research Review, CEPAL Review, Reforma y Democracia, The Oxford Journal of the Welfare States, entre otros. Correo electrónico: juan.bogliaccini@ucu.edu.uy

Rosario Queirolo es profesora del Departamento de Ciencias Sociales y Políticas de la Universidad Católica del Uruguay. Obtuvo su doctorado en Ciencia Política en la University of Pittsburgh (Estados Unidos). Su libro The Success of the Left in Latin America, publicado por University of Notre Dame Press, analiza las razones que llevaron a los latinoamericanos a votar por partidos de izquierda en los 2000s y descarta la idea que este movimiento haya sido el resultado del rechazo popular a las reformas neoliberales. Algunos de sus trabajos han sido publicados en América Latina Hoy, Drugs: Education, Prevention \& Policy, International Journal of Drug Policy, Colombia Internacional, International Journal of Drug Policy, Encyclopedia of Public Administration and Public Policy, Revista Uruguaya de Ciencia Política entre otros. Correo electrónico: rosario.queirolo@ucu.edu.uy 
- 